\title{
CYP1A1 MspI polymorphism and the risk of oral squamous cell carcinoma: Evidence from a meta-analysis
}

\author{
SHANG XIE ${ }^{1}$, CHONGDAI LUO $^{2}$, XIAOFENG SHAN $^{1}$, SHUSHAN ZHAO $^{3}$, JING HE $^{4}$ and ZHIGANG CAI ${ }^{1}$ \\ ${ }^{1}$ Department of Oral and Maxillofacial Surgery, Peking University School and Hospital of Stomatology, Beijing 100081; \\ ${ }^{2}$ Guanghua School and Hospital of Stomatology, Sun Yat-Sen University, Guangzhou, Guangdong 510055; \\ ${ }^{3}$ Department of Orthopaedic Surgery, Xiangya Hospital, Changsha, Hunan $410008 ;{ }^{4}$ State Key Laboratory \\ of Oncologyin South China, Department of Experimental Research, Collaborative Innovation Center \\ for Cancer Medicine, Sun Yat-Sen University Cancer Center, Guangzhou, Guangdong 510060, P.R. China
}

Received September 5, 2014; Accepted January 29, 2016

DOI: $10.3892 /$ mco.2016.768

\begin{abstract}
Numerous case-control studies have investigated whether the CYPIAl gene polymorphism is involved in the occurrence of oral squamous cell carcinoma (OSCC); however, the conclusions are inconsistent. In order to further explore the correlation and obtain a strong conclusion, a meta-analysis was performed to systematically assess the association between the CYPIAI MspI polymorphism and risk of OSCC. In the present meta-analysis, the odds ratios (ORs) and the corresponding 95\% confidence intervals (CIs) were used to assess the association. The statistical analyses were performed with STATA 11.0 software. The heterogeneity was assessed by $\mathrm{Q}$ test and $\mathrm{I}^{2}$ test. The final analysis included 10 studies of 1,505 cases and 1,967 controls. The overall results suggested that the CYPIAI MspI polymorphism was significantly associated with an increased risk of OSCC (CC+TC vs. TT: OR, 1.31; 95\% CI, 1.01-1.70; $\mathrm{P}=0.043$; CC vs. TC+TT: OR, 2.38; 95\% CI, 1.58-3.58; $\mathrm{P}<0.001$; CC vs. TT: OR, 2.52; 95\% CI, 1.60-3.96; $\mathrm{P}<0.001$; and $\mathrm{C}$ vs. T: OR, 1.45 ; 95\% CI, 1.15-1.83; $\mathrm{P}<0.001)$. In a stratified analysis by ethnicity, a statistically significant correlation existed in the Asian population, but not mixed-race and Caucasian populations. In conclusion, despite several limitations, the present meta-analysis established that the CYPlAl MspI
\end{abstract}

Correspondence to: $\mathrm{Dr}$ Jing He, State Key Laboratory of Oncology in South China, Department of Experimental Research, Collaborative Innovation Center for Cancer Medicine, Sun Yat-Sen University Cancer Center, 651 Dongfeng Road East, Guangzhou, Guangdong 510060, P.R. China

E-mail: hejing198374@gmail.com

Professor Zhigang Cai, Department of Oral and Maxillofacial Surgery, Peking University School and Hospital of Stomatology, 22 Zhong Guan Cun South Street, Beijing 100081, P.R. China

E-mail: c2013xs@163.com

Key words: CYP1A1, polymorphism, oral squamous cell carcinoma, meta-analysis polymorphism may be a risk factor for OSCC, particularly among the Asian population.

\section{Introduction}

Oral cancer is one of the most common cancers in the world and causes a considerable problem to global public health due to high mortality rates and disfigurement $(1,2)$. Approximately $90 \%$ of malignant oral neoplasms are oral squamous cell carcinomas (OSCC), followed by adenocarcinoma and, rarely, other types (3). Despite advances in treatment for OSCC, the 5-year survival rate remains poor (4-6). Therefore, investigating the risk factors and developing the early diagnosis for treatment and prevention of OSCC are urgently required.

Epidemiological studies have shown that OSCC is associated with high tobacco use and alcohol consumption (7-9). However, not all individuals with tobacco and alcohol habits develop these fatal diseases, suggesting that individual genetic factors may also be involved in disease etiology. The research results of the human genome project have demonstrated that 99.9\% of the genomes are the same between individuals, with little difference in single nucleotide polymorphisms (SNPs). Therefore, interindividual differences in expression of SNPs may contribute to the variability in the risk towards various types of malignancies, including OSCC. Currently, the published evidence shows that there were significant associations of gene polymorphisms with the susceptibility of numerous cancers, such as GST and CYP1A1 gene polymorphisms with squamous cell carcinoma of the lungs and head and neck cancer, and the 8q24 rsl3281615 polymorphism with the risk of breast cancer (10-15). However, the associations of OSCC with CYPIAl MspI genetic variants are inconsistent (16-25).

Cytochrome P4501A1 (CYP1A1) is a member of the CYP family that participates in the metabolism of xenobiotics and endogenous compounds, encoding for the aryl hydrocarbon hydrolase, which is involved in the activation of polycyclic aromatic hydrocarbon (PAHs) and aromatic amines, and is expressed in oral tissue (26). CYP1A1 is able to activate carcinogenic PAHs and its expression and function are affected by gene polymorphisms, with more attention focused on the 
association of cancer and CYP1A1. According to the previous studies, the CYPIAl gene has several SNPs that may alter the activities of their enzymes and increase carcinogen activation and yield to carcinogenicity. The first allele variants of the CYPlAl gene (CYPIAl*2A or CYP1Al MspI) are the most common polymorphisms, which are a transition from $\mathrm{T}$ to $\mathrm{C}$ in the 3' non-coding region resulting in the introduction of an MspI restriction site and association with an increase in enzyme activity, thus affecting the risks of carcinoma $(27,28)$. The MspI restriction site polymorphism results in three genotypes; wild-type (TT), heterozygous variant (TC) and homozygous variant (CC) (29).

Considering the significance of the CYPIAl MspI polymorphism in the occurrence and development of malignancies, including OSCC, the role of the CYPIAl MspI polymorphism in OSCC patients was systematically evaluated through a meta-analysis.

\section{Materials and methods}

Search strategy. Pubmed, Web of Science, China National Knowledge Infrastructure (CNKI) and WANFANG databases were searched without language limitations, and the last search was updated on May 3, 2014. The CNKI and WANFANG databases provided studies in Chinese and English. The search process was designed to primarily identify all the relevant studies and the search strategies are as follows: (CytochromeP450 1A1 or P4501A1 or CYP1A1 or CYP1A1*2A or MspI or T3801C), (genotype or polymorphism or allele or variant) and (oral squamous cell carcinoma or OSCC or mouth neoplasm or oral cancer or oral carcinoma or oral tumor). The results were screened by two investigators according to the title, key words, abstract and type of study, and irrelevant studies were removed. A manual review of the references cited in the selected studies was undertaken to retrieve studies that may have been missed in the search. Subsequently, the relevant studies were downloaded and further screened to identify the potentially eligible studies. When essential data were not provided in the original studies, every effort was made to contact the authors for confirmation.

Inclusion/exclusion criteria. All the relevant case-control studies were included, irrespective of languages. In the meta-analysis, the following criteria were set and reviewed by two independent investigators: i) Studies should be concerned with the association of the CYPIAl MspI polymorphism with oral squamous cell carcinoma risk, and OSCC cases were histologically confirmed; ii) each trial should be an observational study (case-control or cohort) of human subjects; iii) studies must offer the size of the sample, and the genetic distribution or the original information that can help infer the results; and iv) when multiple studies from a particular research group reported data from overlapping samples, the study reporting the largest dataset was included.

Exclusion criteria included: i) Review studies, editorials or meta-analysis; ii) case reports or lack of case-control study; and iii) studies that estimated the risk of secondary tumors, recurrence or response to treatment. For a conflicting evaluation, an agreement was reached following a discussion. When a consensus could not be attained, another investigator was invited to resolve the dispute and a final result was generated by the majority. All the studies were viewed in accordance with the criteria defined above for further analysis.

Data extraction. All the data were independently reviewed and extracted with a standardized data-collection form by two investigators (Shang Xie and Chongdai Luo). Differences between the investigators were solved by discussion and when necessary, through consultation. The following characteristics were collected from each study: Ethnicity, country, sample size, control source, matching contents, Hardy-Weinberg equilibrium and the gene distribution of cases and controls. When the data were not clear or presented by the author in the publication, contact for further details was attempted.

Quality assessment. The Newcastle-Ottawa scale (NOS) quality evaluation criteria was performed to evaluate the methodological quality of the included studies and those with poor quality were excluded $(30,31)$. The NOS system categorizes into three dimensions, which are selection, comparability and exposure (case-control studies), and the three dimensions included eight items. A star system was used to assess the quality of all the included studies. The NOS ranges from zero (the lowest) to nine (the highest) stars. The assessment was performed independently by two investigators and the discrepancy was resolved by a discussion.

Statistical analysis. All the data management and analysis for the meta-analysis was performed with STATA 11.0 software (Stata Corporation, College Station, TX, USA). The odds ratio (ORs) with corresponding 95\% confidence intervals (CIs) were used to estimate the associations between the CYPIAl MspI polymorphism and OSCC risks. In order to calculate the heterogeneity of the studies, the $\chi^{2}$ test was used and $\mathrm{P}<0.05$ was considered to indicate a statistically significant difference (32). The inconsistency index, $\mathrm{I}^{2}$, was calculated to assess the variation caused by heterogeneity. When the P-value of the heterogeneity test was $>0.10$, the fixed-effects model was performed to calculate the combined OR, which assumed the same homogeneity of effect size across all the studies. When the P-value of the heterogeneity test was $<0.10$, the between-study heterogeneity was considered to indicate a statistically significant difference, and a random effect model was used to estimate the pooled OR. The funnel plot was used to test the underlying publication bias, and the funnel plot asymmetry was estimated by Egger's linear regression (33). Sensitivity analyses were performed to identify the influence of the individual studies on the combined OR. In the analysis, each study was excluded to assess whether stability between the remaining studies was reached.

\section{Results}

Characteristics of included studies. A total of 212 studies were retrieved by the literature search. In total, 171 studies were excluded as they were irrelevant to CYP1Al MspI, OSCC or gene polymorphisms, and were not human studies. Two other potential eligible studies were obtained by screening the references of reviews. Following more detailed evaluations for the remaining 43 potential eligible studies, 


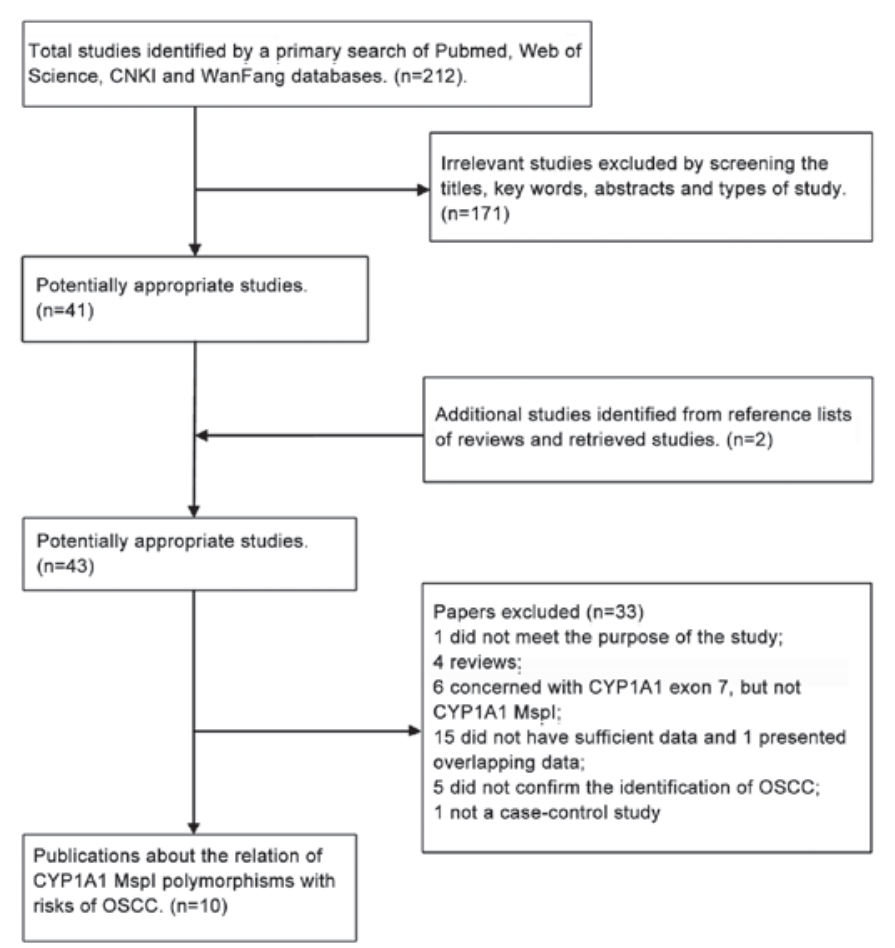

Figure 1. Flow diagram of the included/excluded studies.

one study obtained from references did not meet the purpose of the meta-analysis (34), and four were reviews (26,35-37). Following this, six studies only regarded CYPIAl exon 7, but not CYPIAl MspI (38-43). Another sixteen studies were excluded as one of them presented overlapping data (44) and 15 failed to provide sufficient genotyping data (45-59). In addition, there were five studies excluded as the cases were diagnosed as oral cancer only, and the identification of OSCC was not confirmed (60-64). One study was excluded as the study only contained the cases and lacked the controls (65). Finally, 10 studies conformed to the inclusion criteria and were included in the meta-analysis of CYP1Al MspI (16-25). The search process is shown in Fig. 1.

A database with regard to the information extracted from each included study was established. Summaries of these studies are presented in Table I, which includes the first author, ethnicity, country, number and characteristics of cases and controls, and other necessary information. Of the 10 studies included in the meta-analysis, seven studies were performed in Asian countries, two in American countries and one in European countries. The number of cases and controls in the studies included varied from 38-446 and 81-727, respectively. The frequency of the CYPIAI MspI homozygous variant allele $(\mathrm{C} / \mathrm{C})$ in the cases group varied from $0-30.0 \%$, and $0-10.5 \%$ for the control group.

Results of quality assessment. According to the NOS system, all the included case-control studies were awarded a maximum of four stars in selection, two stars in comparability and three stars in exposure. The results of the assessment for the included studies ranged from six to eight stars (Table I), indicating that all the included studies were moderate-high qualities.

Test of heterogeneity and quantitative synthesis. A heterogeneity analysis was performed of the dominant (CC+TC vs. TT),

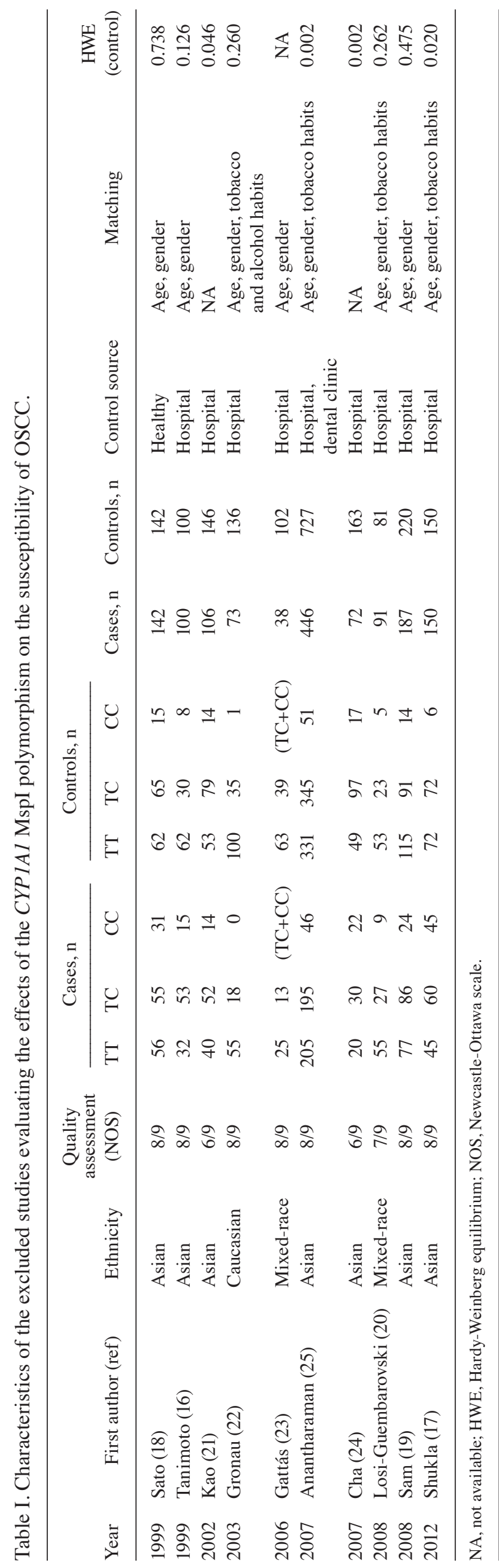




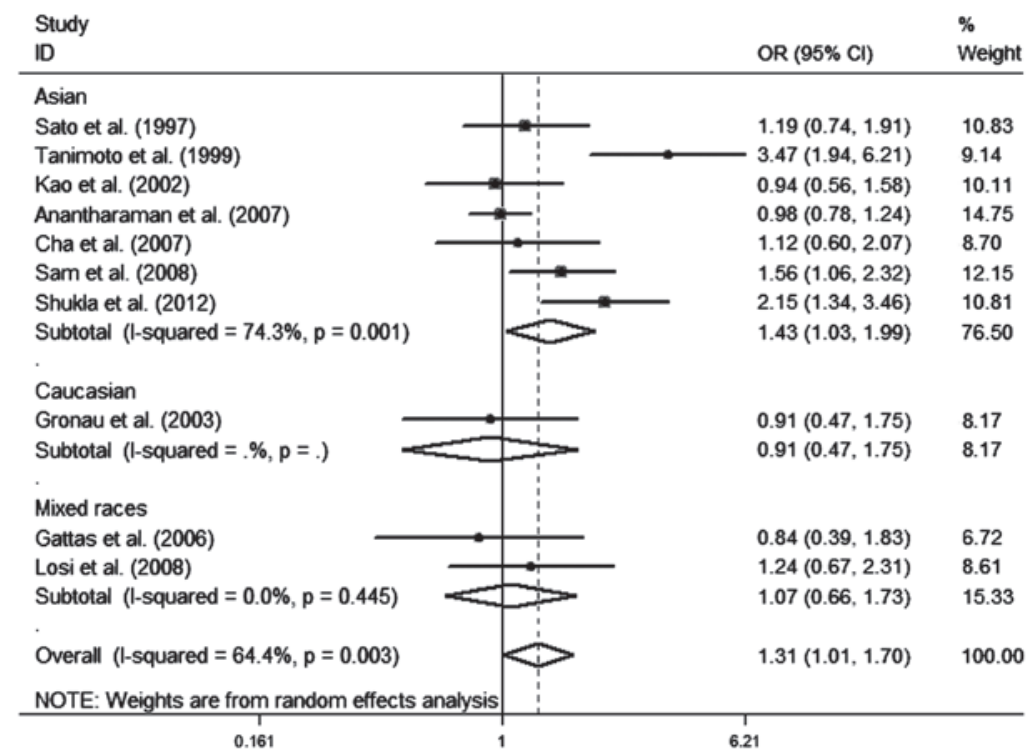

Figure 2. Forest plot of the association between the CYP1A1 MspI polymorphism with the risk of OSCC (dominant model: CC+TC vs. TT; stratified by ethnicity). OSCC, oral squamous cell carcinoma.

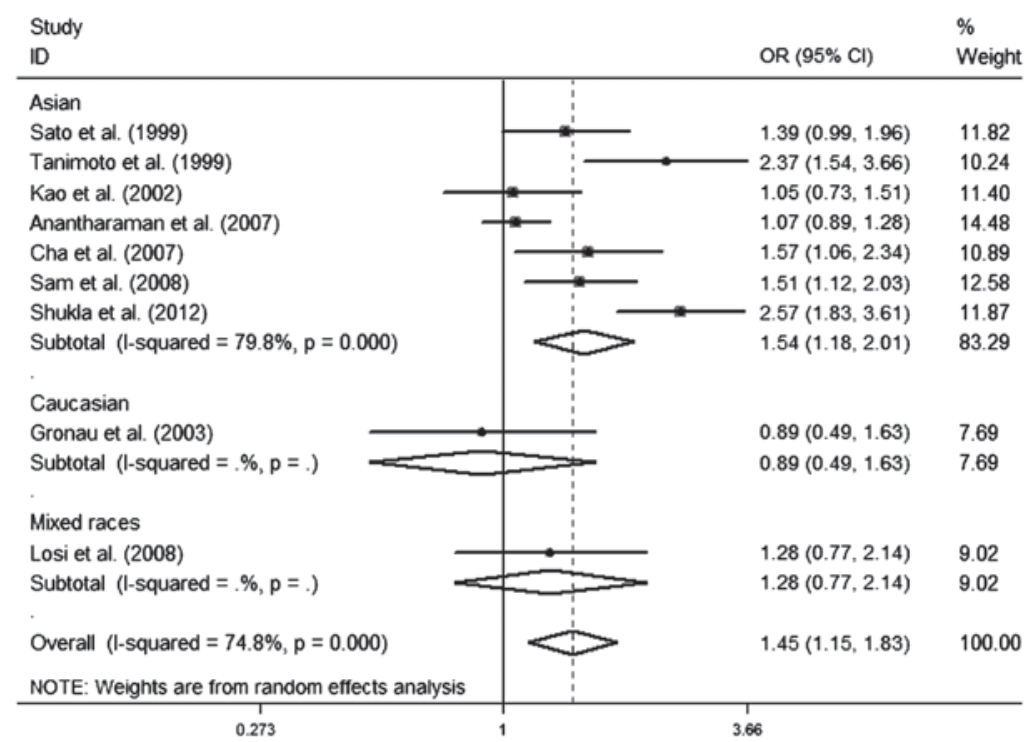

Figure 3. Forest plot of the association between the $\mathrm{C}$ allele of the CYP1A1 MspI polymorphism and the risk of OSCC (allele model: C vs. T, stratified by ethnicity). OSCC, oral squamous cell carcinoma.

recessive (CC vs. TC+TT) and additive models (CC vs. TT), and the results are shown in Table II. Owing to the overall heterogeneity observed in the dominant $(\mathrm{CC}+\mathrm{TC}$ vs. TT: $\left.\mathrm{I}^{2}=64.4 \%, \mathrm{P}=0.003\right)$, recessive $\left(\mathrm{CC}\right.$ vs. TC+TT: $\mathrm{I}^{2}=57.9 \%$, $\mathrm{P}=0.015$ ) and additive models (CC vs. TT: $\mathrm{I}^{2}=61.0 \%, \mathrm{P}=0.009$ ), random-effect models were used to synthesize the data, respectively (Table II). The overall results suggested that the CYPlAl gene variants ( $\mathrm{TC}+\mathrm{CC}$ or $\mathrm{CC}$ ) have an increased risk of OSCC compared to those individuals with the positive homozygous carriers (TT). In order to further explore the observed heterogeneity, subgroup analyses were performed by ethnicity and 10 studies were divided into three subgroups: the Asian, Caucasian and mixed-race groups. However, the heterogeneity remained in the Asian population, but not in the mixed-race and Caucasian populations. For ethnicity, a significant increased risk was associated with the genetic variants among the Asian population, while no associations were found among the mixed ethnic and Caucasian populations (Fig. 2 and Table II).

As for the $\mathrm{C}$ and $\mathrm{T}$ allele of CYPIAl MspI, the results of the heterogeneity test and quantitative synthesis of $\mathrm{C}$ vs. $\mathrm{T}$ model, the pooled OR, 1.447 ; 95\% CI, 1.146-1.827; $\mathrm{I}^{2}=74.8 \%$; $\mathrm{P}_{\mathrm{Q} \text { - test }}=0.000$; and $\mathrm{P}<0.05$ (Fig. 3 ) suggested that the $\mathrm{C}$ allele was significantly associated with an increased OSCC risk.

Publication bias analysis. The Begg's funnel plot was used to assess the possible publication bias. The Egger's linear regression is for the quantitative evaluation of the meta-analysis funnel plot symmetry and the results were as follows: i) CC+TC vs. TT model: Begg's test, $\mathrm{P}=0.858>0.05$ and Egger's linear 
regression test: $\mathrm{t}=0.85, \mathrm{P}=0.419>0.05$; ii) $\mathrm{CC}$ vs. $\mathrm{TC}+\mathrm{TT}$ model: Begg's test, $\mathrm{P}=0.711>0.05$ and Egger's linear regression test: $\mathrm{t}=1.05, \mathrm{P}=0.335>0.05$; iii) $\mathrm{CC}$ vs. TT model: Begg's test, $\mathrm{P}=0.266>0.05$ and Egger's linear regression test: $\mathrm{t}=1.20$, $\mathrm{P}=0.276>0.05$; and iv) $\mathrm{C}$ vs. $\mathrm{T}$ allele model: Begg's test, $\mathrm{P}=1.000>0.05$ and Egger's linear regression test: $\mathrm{t}=0.99$, $\mathrm{P}=0.354>0.05$. The data of the four models indicated that the funnel plots were symmetrical for all.

Sensitivity analysis. In order to assess the stability of the results and reflect the influence of each study on the pooled ORs, sensitivity analysis was performed by excluding each case-control study individually. All the estimates were included between the lower and upper CI limits, suggesting the stability of the results in the meta-analysis.

\section{Discussion}

Oral cancer is cancer of the mouth, including squamous cell carcinoma, adenocarcinoma and verrucous carcinoma. Different histopathological types of cancers may have different genetic susceptibilities, such as CYPIA1 MspI polymorphism being a risk factor of squamous cell carcinoma of the lung, but varies in different histological types $(13,66)$. Therefore, it is more reasonable to separately evaluate the association of gene polymorphisms with OSCC, oral adenocarcinoma and other cancer types.

To the best of our knowledge, this is the first meta-analysis to assess the association between the CYPIAl MspI genetic variants and risks of OSCC. Although there are two previous meta-analyses $(67,68)$ regarding the CYPIAl MspI polymorphism and oral cancer, the results did not involve the single histopathological type and therefore cannot represent the association of CYPIAl MspI with the risks of OSCC. Oral cancer is known to include different histological types, including squamous cell carcinoma and adenocarcinoma, which may yield to different susceptibilities of cancer. Therefore, the previous studies' results may regard all types of oral carcinoma for only one selection. To obtain a powerful conclusion regarding the risks of OSCC and CYPIAI MspI polymorphism, a systematical meta-analysis was performed in the present study.

In the present meta-analysis, for the overall data the results of ORs and 95\% CIs showed that the C allele of CYP1Al MspI played a significant role in the carcinogenesis process resulting in OSCC, and as for the genotypes, $\mathrm{CC}$ and $\mathrm{CT}+\mathrm{CC}$ were identified as risk factors for developing OSCC. All the results indicated that the CYPIAl MspI polymorphism may increase the risks of OSCC. The heterogeneity among studies was observed in the dominant, recessive, additive and $C$ versus $\mathrm{T}$ allele models, respectively. Following the subgroup analysis by ethnicity, the heterogeneity was not removed indicating that other factors, such as age, gender, country, source of controls, lifestyle, social status, smoking and alcohol habits, may also yield to heterogeneities.

In the subgroup analysis by ethnicity, a key association between the CYPIAl MspI polymorphism and risks of OSCC in the Asian population was confirmed in all four models, but not in the mixed-race and Caucasian populations, suggesting that the CYP1Al MspI gene variants may increase the OSCC susceptibility in the Asian population. The differences may be 
attributed to different ethnicities sharing different gene-gene and gene-environmental backgrounds. Nevertheless, the conclusion regarding the mixed-race and Caucasian populations is not of a sufficient power for the few studies and subjects.

Publication biases were evaluated by funnel plots and their symmetries, and were further assessed by Begg's test and Egger's linear regression tests, respectively. No clear biases were observed, indicating that the publication may yield to little effects on the results. The sensitivity analysis showed that the importance of the corresponding pooled ORs was not significantly changed, suggesting that the pooled ORs were stable.

However, several limitations should be addressed. First of all, the original studies included data regarding the Asian, Caucasian and mixed-race populations, and only one study regarding Caucasian and two mixed-race populations. Secondly, a subgroup analysis was performed by ethnicity, but the other factors, such as gender, age, source of control and country, were not performed due to data limitations. Thirdly, the Asian population included India, Japan and China, but other Asian countries were not included. Fourthly, heterogeneity existed, which may weaken the reliability of the conclusions. In view of these limitations, the results should be considered with caution.

Overall, despite several limitations the results of the present analysis showed a clear association between the CYP1A1 MspI polymorphism and OSCC risk, particularly among the Asian population. Future studies focusing on the CYPIAl MspI polymorphism containing larger sample sizes and well-matched criteria are required to improve the credibility of the conclusions.

\section{Acknowledgements}

The present study was supported by Special Financial Grants from the China Postdoctoral Science Foundation (no. 2014T70836) and the National Natural Science Foundation of China (nos. 81371162, 8110762 and 30973336). The authors acknowledge Dr Wenjie Li (Department of Oral Health Sciences, School of Dentistry, University of Washington, Seattle, WA, USA) and Dr Xinchen Yang (Department of Oral Rehabilitation, Faculty of Dentistry, University of Hong Kong, Sai Ying Pun, Hong Kong, China) for assistance in collecting the relevant studies.

\section{References}

1. Kademani D: Oral cancer. Mayo Clin Proc 82: 878-887, 2007.

2. Petersen PE: Oral cancer prevention and control - the approach of the World Health Organization. Oral Oncol 45: 454-460, 2009.

3. Barnes L, Eveson JW, Reichart P and Sidransky D (eds): Tumours of the oral cavity and oropharynx. In: World Health Organization Classification of Tumours: Pathology and Genetics of Head and Neck Tumours. Vol 9. 3rd edition. IARC Press, Lyon, France, pp166-210, 2005.

4. Chen GS and Chen $\mathrm{CH}$ : A study on survival rates of oral squamous cell carcinoma. Kaohsiung J Med Sci 12: 317-325, 1996 (In Chinese)

5. Lim YC and Choi EC: Surgery alone for squamous cell carcinoma of the oral cavity: survival rates, recurrence patterns, and salvage treatment. Acta Otolaryngol 128: 1132-1137, 2008

6. Lo Muzio L, Campisi G, Farina A, et al P-cadherin expression and survival rate in oral squamous cell carcinoma: an immunohistochemical study. BMC Cancer 5: 63, 2005.
7. Blot WJ, McLaughlin JK, Winn DM, et al: Smoking and drinking in relation to oral and pharyngeal cancer. Cancer Res 48: 3282-3287, 1988.

8. Figuero Ruiz E, Carretero Peláez MA, Cerero Lapiedra R, Esparza Gómez G and Moreno López LA: Effects of the consumption of alcohol in the oral cavity: relationship with oral cancer. Med Oral 9: 14-23, 2004.

9. Johnson N: Tobacco use and oral cancer: a global perspective. J Dent Educ 65: 328-339, 2001

10. Zhang Y, Ni Y, Zhang H, Pan Y, Ma J and Wang L: Association between GSTM1 and GSTT1 allelic variants and head and neck squamous cell cancinoma. PLoS One 7: e47579, 2012.

11. Wang Y, Yang H, Li L and Wang H: Glutathione S-transferase $\mathrm{T} 1$ gene deletion polymorphism and lung cancer risk in Chinese population: a meta-analysis. Cancer Epidemiol 34: 593-597, 2010.

12. Pei YL, Zhang HL and Han HG: Polymorphism of $8 \mathrm{q} 24$ rsl3281615 and breast cancer risk: a meta-analysis. Tumor Biol 34: 421-428, 2013.

13. Ji YN, Wang Q and Suo LJ: CYP1A1 Ile462Val polymorphism contributes to lung cancer susceptibility among lung squamous carcinoma and smokers: a meta-analysis. PloS One 7: e43397, 2012.

14. Hashibe M, Brennan P, Strange RC, et al: Meta- and pooled analyses of GSTM1, GSTT1, GSTP1, and CYP1A1 genotypes and risk of head and neck cancer. Cancer Epidemiol Biomarkers Prev 12: 1509-1517, 2003

15. Feng X, Zhou H-F, Zheng B-S, Shi J-J, Luo C and Qin J-J: Association of glutathione S-transferase P1 gene polymorphism with the histological types of lung cancer: a meta-analysis. Mol Biol Rep 40: 2439-2447, 2013.

16. Tanimoto $\mathrm{K}$, Hayashi $\mathrm{S}$, Yoshiga $\mathrm{K}$ and Ichikawa $\mathrm{T}$ : Polymorphisms of the CYP1A1 and GSTM1 gene involved in oral squamous cell carcinoma in association with a cigarette dose. Oral Oncol 35: 191-196, 1999.

17. Shukla D, Dinesh Kale A, Hallikerimath S, Vivekanandhan S and Venkatakanthaiah Y: Genetic polymorphism of drug metabolizing enzymes (GSTM1 and CYP1A1) as risk factors for oral premalignant lesions and oral cancer. Biomed Pap Med Fac Univ Palacky Olomouc Czech Repub 156: 253-259, 2012.

18. Sato M, Sato T, Izumo T and Amagasa T: Genetic polymorphism of drug-metabolizing enzymes and susceptibility to oral cancer. Carcinogenesis 20: 1927-1931, 1999.

19. Sam SS, Thomas V, Reddy KS, Surianarayanan G and Chandrasekaran A: CYP1A1 polymorphisms and the risk of upper aerodigestive tract cancers in an indian population. Head Neck 30: 1566-1574, 2008.

20. Losi-Guembarovski R, Cólus IM, De Menezes RP, et al: Lack of association among polymorphic xenobiotic-metabolizing enzyme genotypes and the occurrence and progression of oral carcinoma in a brazilian population. Anticancer Res 28: 1023-1028, 2008.

21. Kao SY, Wu CH, Lin SC, et al: Genetic polymorphism of cytochrome P4501A1 and susceptibility to oral squamous cell carcinoma and oral precancer lesions associated with smoking/betel use. J Oral Pathol Med 31: 505-511, 2002.

22. Gronau S, Koenig-Greger D, Jerg M and Riechelmann H: GSTM1 enzyme concentration and enzyme activity in correlation to the genotype of detoxification enzymes in squamous cell carcinoma of the oral cavity. Oral Dis 9: 62-67, 2003.

23. Gattás GJ, de Carvalho MB, Siraque MS, et al: Genetic polymorphisms of CYP1A1, CYP2E1, GSTM1, and GSTT1 associated with head and neck cancer. Head Neck 28: 819-826, 2006.

24. Cha IH, Park JY, Chung WY, Choi MA, Kim HJ and Park KK: Polymorphisms of CYP1A1 and GSTM1 genes and susceptibility to oral cancer. Yonsei Med J 48: 233-239, 2007.

25. Anantharaman D, Chaubal PM, Kannan S, Bhisey RA and Mahimkar MB: Susceptibility to oral cancer by genetic polymorphisms at CYP1A1, GSTM1 and GSTT1 loci among indians: tobacco exposure as a risk modulator. Carcinogenesis 28 : 1455-1462, 2007.

26. Bartsch H, Nair U, Risch A, Rojas M, Wikman H and Alexandrov K: Genetic polymorphism of CYP genes, alone or in combination, as a risk modifier of tobacco-related cancers. Cancer Epidemiol Biomarkers Prev 9: 3-28, 2000.

27. Petersen DD, McKinney CE, Ikeya K, et al: Human CYP1A1 gene: cosegregation of the enzyme inducibility phenotype and an RFLP. Am J Hum Genet 48: 720-725, 1991.

28. Landi MT, Bertazzi PA, Shields PG, et al: Association between CYP1A1 genotype, mRNA expression and enzymatic activity in humans. Pharmacogenetics 4: 242-246, 1994. 
29. Zhou SF, Liu JP and Chowbay B: Polymorphism of human cytochrome P450 enzymes and its clinical impact. Drug Metab Rev 41: 89-295, 2009.

30. Wells GA, Shea B, O'Connell D, Peterson J, Welch V, Losos M and Tugwell P: The Newcastle-Ottawa scale (NOS) for assessing the quality of nonrandomised studies in meta-analyses. http://www. ohri.ca/programs/clinical_epidemiology/oxford.asp. Accessed February 3, 2016.

31. Stang A: Critical evaluation of the Newcastle-Ottawa scale for the assessment of the quality of nonrandomized studies in meta-analyses. Eur J Epidemiol 25: 603-605, 2010.

32. Cochran WG: The combination of estimates from different experiments. Biometrics 10: 101-129, 1954

33. Egger M, Davey Smith G, Schneider M and Minder C: Bias in meta-analysis detected by a simple, graphical test. BMJ 315: 629-634, 1997.

34. Matthias C, Bockmühl U, Jahnke V, Harries LW, Wolf CR, et al: The glutathione S-transferase GSTP1 polymorphism: effects on susceptibility to oral/pharyngeal and laryngeal carcinomas. Pharmacogenetics 8: 1-6, 1998 .

35. Khlifi R, Messaoud O, Rebai A and Hamza-Chaffai A: Polymorphisms in the human cytochrome P450 and arylamine N-Acetyltransferase: susceptibility to head and neck cancers. Biomed Res Int 2013: 582768, 2013.

36. Liu L, Wu G, Xue F, et al: Functional CYP1A1 genetic variants, alone and in combination with smoking, contribute to development of head and neck cancers. Eur J Cancer 49: 2143-2151, 2013.

37. Ruwali M and Parmar D: Association of functionally important polymorphisms in cytochrome P450s with squamous cell carcinoma of head and neck. Indian J Exp Biol 48: 651-665, 2010

38. Amtha R, Ching CS, Zain R, et al: GSTM1, GSTT1 and CYP1A1 polymorphisms and risk of oral cancer: a case-control study in Jakarta, Indonesia. Asian Pac J Cancer Prev 10: 21-26, 2009.

39. Buch SC, Nazar-Stewart V, Weissfeld JL and Romkes M: Case-control study of oral and oropharyngeal cancer in whites and genetic variation in eight metabolic enzymes. Head Neck 30 : $1139-1147,2008$

40. Katoh T: Application of molecular biology to occupational health field - the frequency of gene polymorphism of cytochrome P450 1A1 and glutathione S-transferase M1 in patients with lung, oral and urothelial cancer. J UOEH 17: 271-278, 1995 (In Japanese).

41. Katoh T, Kaneko S, Kohshi K, et al: Genetic polymorphisms of tobacco- and alcohol-related metabolizing enzymes and ora cavity cancer. Int J Cancer 83: 606-609, 1999.

42. Marques CF, Koifman S, Koifman RJ, Boffetta P, Brennan P and Hatagima A: Influence of cyp1a1, CYP2E1, GSTM3 and NAT2 genetic polymorphisms in oral cancer susceptibility: results from a case-control study in Rio de Janeiro. Oral Oncol 42: 632-637, 2006.

43. Sugimura T, Kumimoto H, Tohnai I, et al: Gene-environment interaction involved in oral carcinogenesis: molecular epidemiological study for metabolic and DNA repair gene polymorphisms. J Oral Pathol Med 35: 11-18, 2006.

44. Shukla D, Kale AD, Hallikerimath S, Yerramalla V, Subbiah V and Mishra S: Association between GSTM1 and CYP1A1 polymorphisms and survival in oral cancer patients. Biomed Pap Med Fac Univ Palacky Olomouc Czech Repub 157: 304-310, 2013.

45. Anantharaman D, Samant TA, Sen S and Mahimkar MB Polymorphisms in tobacco metabolism and DNA repair genes modulate oral precancer and cancer risk. Oral Oncol 47: 866-872, 2011.

46. Boccia S, Cadoni G, Sayed-Tabatabaei FA, et al: CYP1A1, CYP2E1, GSTM1, GSTT1, EPHX1 exons 3 and 4, and NAT2 polymorphisms, smoking, consumption of alcohol and fruit and vegetables and risk of head and neck cancer. J Cancer Res Clin Oncol 134: 93-100, 2008.

47. Canova C, Richiardi L, Merletti F, et al: Alcohol, tobacco and genetic susceptibility in relation to cancers of the upper aerodigestive tract in northern Italy. Tumori 96: 1-10, 2010.

48. Cury NM, Russo A, Galbiatti ALS, et al: Polymorphisms of the CYP1A1 and CYP2E1 genes in head and neck squamous cell carcinoma risk. Mol Biol Rep 39: 1055-1063, 2012.

49. Ko Y, Abel J, Harth V, et al: Association of CYP1B1 codon 432 mutant allele in head and neck squamous cell cancer is reflected by somatic mutations of p53 in tumor tissue. Cancer Res 61: 4398-4404, 2001.
50. Olivieri EH, da Silva SD, Mendonca FF, et al: CYP1A2*1C, CYP2E1*5B, and GSTM1 polymorphisms are predictors of risk and poor outcome in head and neck squamous cell carcinoma patients. Oral Oncol 45: e73-e79, 2009.

51. Ramadas K, Ramachandran S, Muwonge R and Pillai MR Tumor progression in the oral cavity: The significance of genetic polymorphisms in CYP1A1, GST M1 and XRCC1 in a south Indian population. Oral Oncol Supplement (Suppl 3): 69-69, 2009.

52. Sabitha K, Reddy MV and Jamil K: Smoking related risk involved in individuals carrying genetic variants of CYP1A1 gene in head and neck cancer. Cancer Epidemiol 34: 587-592, 2010.

53. Sam SS, Thomas V, Reddy KS, Surianarayanan G and Chandrasekaran A: Gene-gene interactions of drug metabolizing enzymes and transporter protein in the risk of upper aerodigestive tract cancers among indians. Cancer Epidemiol 34: 626-633, 2010.

54. Sam SS, Thomas V, Reddy KS, Surianarayanan G and Chandrasekaran A: Gene-environment interactions associated with CYP1A1 Mspi and GST polymorphisms and the risk of upper aerodigestive tract cancers in an indian population. J Cancer Res Clin Oncol 136: 945-951, 2010.

55. Sharma R, Ahuja M, Panda NK and Khullar M: Combined effect of smoking and polymorphisms in tobacco carcinogen-metabolizing enzymes CYP1A1 and GSTM1 on the head and neck cancer risk in North Indians. DNA Cell Biol 29: 441-448, 2010.

56. Sharma R, Ahuja M, Panda NK and Khullar M: Interactions among genetic variants in tobacco metabolizing genes and smoking are associated with head and neck cancer susceptibility in North Indians. DNA Cell Biol 30: 611-616, 2011

57. Sharma R, Panda NK and Khullar M: Hypermethylation of carcinogen metabolism genes, CYP1A1, CYP2A13 and GSTM1 genes in head and neck cancer. Oral Dis 16: 668-673, 2010.

58. Singh AP, Shah PP, Ruwali M, Mathur N, Pant MC and Parmar D: Polymorphism in cytochrome P4501 A1 is significantly associated with head and neck cancer risk. Cancer Invest 27: 869-876, 2009.

59. Yadav SS, Ruwali M, Pant MC, Shukla P, Singh RL and Parmar D: Interaction of drug metabolizing cytochrome P450 2D6 poor metabolizers with cytochrome P450 2C9 and 2C19 genotypes modify the susceptibility to head and neck cancer and treatment response. Mutat Res 684: 49-55, 2010.

60. Chatterjee S, Dhar S, Sengupta B, et al: Polymorphisms of CYP1A1, GSTM1 and GSTT1 loci as the genetic predispositions of oral cancers and other oral pathologies: tobacco and alcohol as risk modifiers. Indian J Clin Biochem 25: 260-272, 2010.

61. Cordero K, Espinoza I, Caceres D, et al: Oral cancer susceptibility associated with the CYP1A1 and GSTM1 genotypes in Chilean individuals. Oncol Lett 1: 549-553, 2010.

62. Guo L, Zhang C, Shi S and Guo X: Correlation between smoking and the polymorphisms of cytochrome P450 1A1-Msp I and glutathione s-transferase T1 genes and oral cancer. Hua Xi Kou Qiang Yi Xue Za Zhi 30: 187-191, 2012 (In Chinese).

63. Masood N, Kayani MA, Malik FA, Mahjabeen I, Baig RM and Faryal R: Genetic variations in carcinogen metabolizing genes associated with oral cancer in pakistani population. Asian Pac J Cancer Prev 12: 491-495, 2011.

64. Matthias C, Bockmühl U, Jahnke V, et al: Polymorphism in cytochrome P450 CYP2D6, CYP1A1, CYP2E1 and glutathione S-transferase, GSTM1, GSTM3, GSTT1 and susceptibility to tobacco-related cancers: studies in upper aerodigestive tract cancers. Pharmacogenetics 8: 91-100, 1998.

65. Chatterjee S, Chakrabarti S, Sengupta B, et al: Prevalence of CYP1A1 and GST polymorphisms in the population of northeastern India and susceptibility of oral cancer. Oncol Res 17: 397-403, 2009.

66. Zhan P, Wang Q, Qian Q, Wei S-Z and Yu L-K: CYP1A1 MspI and exon7 gene polymorphisms and lung cancer risk: an updated meta-analysis and review. J Exp Clin Cancer Res 30: 99, 2011.

67. Zhuo W, Wang Y, Zhuo X, et al: CYP1A1 and GSTM1 polymorphisms and oral cancer risk: association studies via evidence-based meta-analyses. Cancer Invest 27: 86-95, 2009.

68. Zhuo X, Zhao H, Chang A, et al: Quantitative assessment of CYP1A $1 * 2 \mathrm{~A}$ variations with oral carcinoma susceptibility: evidence from 1,438 cases and 2,086 controls. Cancer Invest 30: $552-559,2012$. 